\title{
SUL TERREMOTO PROFONDO DELLE ISOLE EOLIE DEL 23 NOVEMBRE 1954
}

\author{
Antonino Girlanda
}

E accertato che la zona del basso Tirreno, comprendente il gruppo delle Eolie con l'isola di Ustica e parzialmente limitata dall'arco tracciato dalla costa occidentale della Calabria e dalla costa settentrionale della Sicilia, è anche sede di centri sismici profondi. Dagli studi microsismici fino ad oggi eseguiti risulterebbe che tali centri, la cui profondità è compresa tra 200 e $300 \mathrm{~km}$., si dispongono lungo un arco parallelo all'arco costituito dall'Appennino calabro-campano, cioe lungo la fascia vulcanica attiva che comprende l'Etna, lo Stromboli ed il Vesuvio.

Alcune caratteristiche della zona (vulcanismo attivo o recentemente spento, notevoli profondità marine, anomalia isostatica positiva) la rendono, sotto certi aspetti, somigliante ad alcuni tratti dell'arco circum-pacifico, che, secondo l'elaborazione statistica di Gutenberg e Richter $(1)$, è sede del $90 \%$ ca. dei terremoti con profondità compresa tra 60 e $300 \mathrm{~km}$.

Pur essendo definitivamente stabilita, nella zona, l'esistenza di ipocentri profondi, non altrettanto definitivamente risulta confermata l'esistenza, nella zona stessa, di una grande anomalia nella propagazione delle onde di dilatazione e di distorsione. Tale anomalia fu, com noto, sospettata in seguito ad una determinazione di Demetrescu, fondata su dati microsismici, delle coordinate spazio-temporali dell'ipocentro del terremoto del 13 aprile $1938\left(\lambda_{0}=15^{\circ} 05^{\prime} E ; \varphi_{0}=39^{\circ} 34^{\prime} \mathrm{N}\right.$; $h=312 \mathrm{~km}$.; $\left.t_{0}=03^{\mathrm{h}} 45^{\mathrm{m}} 56^{*}\right)$, dalla quale consegue un notevole anticipo nei tempi di registrazione delle $P_{n}$ ed $S_{\mathrm{n}}$ nelle stazioni più vicine alla zona epicentrale. Una conferma in tal senso è stata ottenuta da Di Filippo $(2)$ in uno studio sul terremoto del 16 marzo 1941 $\left(\lambda_{o}=12^{\circ} 07^{\prime} 23^{\prime \prime} E: \varphi_{0}=38^{\prime \prime} 26^{\prime} 19^{\prime \prime} N ; h=85 \mathrm{~km}\right.$; $\left.t_{0}=17^{\mathrm{L}} 07^{\mathrm{m}} 23^{\mathrm{s}}, 5\right)$, sebbene gli anticipi osservati da quest'ultimo Autore siano di minore entità.

D'altra parte, da una ricerca da me effettuata sul terremoto profondo del 26 dicembre $1952\left(\lambda_{0}=15^{\circ} 32^{\prime}, 8 E: \varphi_{0}=39^{\circ} 46^{\prime}, 3 N ; h=265\right.$ 
$\mathrm{km}$; $t_{0}=23^{\mathrm{h}} 55^{\mathrm{m}} 55^{\mathrm{s}}, 6$ ), nonostante siano state escluse dal calcolo delle coordinate spazio-temporali dell'ipocentro i dati delle stazioni con distanza inferiore a $] 000 \mathrm{~km}$., risulta che anche le stazioni più vicine non hanno dato luogo a scarti, tra tempi osservati e tempi calcolati in base alle dromocrone di Jeffreys e Bullen (J-B), che non siano contenibili nei limiti degli errori sperimentali (").

I risultati fino ad oggi ottenuti, dato il loro carattere nettamente contrastante, non permettono di decidere sull'esistenza o meno dell'anomalia su indicata.

Ritengo quindi non del tutto priva di interesse la presente ricerca dedicata ad un nuovo movimento sismico di origine profonda verificatosi nella zona delle isole Eolie il 23 novembre 1954 e registrato a Messina alle $13^{\text {h }} 00^{\mathrm{m}} 36^{\mathrm{s}}, 3$.

TABella I

\begin{tabular}{|c|c|c|c|c|c|}
\hline STAZIONI & $\Delta$ & $\begin{array}{c}T_{p} \\
\text { Tempi di } \\
\text { registrazione }\end{array}$ & $\begin{array}{l}t_{p} \\
\text { (C) }\end{array}$ & $\begin{array}{l}t_{p} \\
(O)\end{array}$ & $\mathrm{O}-\mathrm{C}$ \\
\hline Reggio Calabria & $0^{\circ}, 8265$ & $13 \mathrm{~h} 00^{\mathrm{m}} 36^{\mathrm{s}}$ & $00^{\mathrm{m}} 34^{\mathrm{s}}, 4$ & $00^{\mathrm{m}} 34^{\mathrm{s}}$ & $-0 n, 4$ \\
\hline Messina & 0,7171 & 36,3 & 33,9 & 34,3 & $\therefore 0,4$ \\
\hline Taranto & 2,6663 & 46,0 & 49,4 & 42 & $-7,4$ \\
\hline Rocca di Papa & 3,5352 & 0107 & 58,9 & 0103 & $+4,1$ \\
\hline Roma & 3,7346 & 09 & $01 \quad 01,3$ & 05 & $+3,7$ \\
\hline Firenze Xim. & 5,8219 & 33 & 26,6 & 29 & $+2,9$ \\
\hline Prato & 5,9691 & 36 & 28,4 & 32 & $-3,6$ \\
\hline Atene & 7,0406 & 42,4 & 41,9 & 38,4 & $-3,5$ \\
\hline Trieste & 7,0813 & 50 & 42,4 & 46 & $+3,6$ \\
\hline Salò & 7,6893 & 55 & 50,1 & 51 & $-0,9$ \\
\hline Chur & 9,1067 & 0215,6 & $02 \quad 08,1$ & $02 \quad 11,6$ & $\div 3,5$ \\
\hline Wien & 9,7109 & 23 & 15,8 & 19 & $+3,2$ \\
\hline Zürich & 9,8736 & 25,1 & 17,8 & 21,1 & $+3,3$ \\
\hline Neuchâtel & 10,1748 & 28,5 & 21,6 & 24,5 & $+2,9$ \\
\hline Besançon & 10,7849 & 36 & 29,3 & 32,0 & $+2,7$ \\
\hline Stüttgart & 10,9431 & 38,5 & 31,3 & 34,5 & $+3,2$ \\
\hline Istanbul & 11,2448 & 40,5 & 35,1 & 36,5 & $+1,4$ \\
\hline Strasbourg & 11,2020 & 42 & 34,6 & 38 & +. 3,4 \\
\hline Clermont & 11,2414 & 44 & 35,1 & 40 & $+4,9$ \\
\hline Praha & 11,4712 & 48 & 37,9 & 44 & $+6,1$ \\
\hline Paris & 13,5335 & $03 \quad 10,5$ & 0303,5 & 0306,5 & $+3,0$ \\
\hline Uccle & 14,2594 & $19^{\circ}$ & 12,4 & 15 & $+2,6$ \\
\hline Helwan & 16,2042 & 41 & 35,4 & 37 & $\begin{array}{r}-1,6 \\
-1,6\end{array}$ \\
\hline Ksara & 17,6647 & 56 & 51,2 & 52 & $+0,8$ \\
\hline Tamanrasset & 17,6434 & 56 & 50,9 & 52 & $+1,0$ \\
\hline Rathfarnham & 20,6456 & 0432 & $04 \quad 21,3$ & $04 \quad 28$ & $+6,7$ \\
\hline Uppsala & 21,3541 & 33 & 28,2 & 29 & $-0,8$ \\
\hline Kiruna & 29,4597 & 0547 & $05 \quad 42,8$ & $05 \quad 43$ & $+0,2$ \\
\hline
\end{tabular}


Da un esame dei tempi di registrazione delle $P_{n}$ elencati nel Bollettino del B.C.I.S. ho notato che, assumendo come coordinate spaziotemporali quelle riportate nello stesso Bollettino, i tempi di tragito osservati non coincidono in genere con gli analoghi tempi calcolati servendosi delle dromocrone di J-B. Più precisamente ho notato che, escluse le stazioni di Messina, Reggio Calabria, Salo, Ksara, Uppsala e Kiruna, nelle quali si hanno scarti che non superano il secondo, le differenze $\mathrm{O}$-C sono sistematicamente positive; solo Taranto ed Atene danno luogo a notevoli scarti negativi (tabella I). Tale fatto è certamente indizio di una valutazione non sufficientemente approssimata delle coordinate spazio-temporali.

A scopo orientativo ho calcolato le correzioni da apportare alle costanti ipocentrali determinate dal B.C.I.S., servendomi degli inizi delle $P_{n}$ osservati in 14 stazioni e riportati nel grià citato Bollettino. Ho escluso dalla determinazione i dati delle stazioni con distanza epicentrale inferiore a $1000 \mathrm{~km}$. per evitare le possibili influenze derivanti dalla indicata eventuale anomalia nella velocita di propagazione delle $P_{n}$ nelle vicinanze della zona epicentrale. Servendomi del noto metodo statistico di Caloi $\left({ }^{+}\right)$, assumendo come valori di partenza i seguenti

$$
\begin{aligned}
& \left(\lambda_{0}\right)=+14^{\circ} 48^{\prime}, \\
& \left(\varphi_{0}\right)=+38^{\prime \prime} 36^{\prime} \quad \text { (lat. geografica), } \\
& \left(\Phi_{0}\right)=+38^{\prime \prime} 24^{\prime} 42^{\prime \prime}, 101 \quad \text { (lat. geocentrica), } \\
& (h)=3 R 10^{-2}+33 \mathrm{~km} \quad(R=6338 \mathrm{~km}), \\
& \left(t_{0}\right)=13^{\mathrm{h}} 00^{\mathrm{m}} 05^{\mathrm{s}}, 411,
\end{aligned}
$$

ed utilizzando gli elementi dedotti dai dati delle stazioni elencate nella tabella II, ho ottenuto le seguenti correzioni:

$$
\begin{aligned}
& \delta \lambda_{0}=+05^{\prime} 41^{\prime \prime}, 916+02^{\prime} 04^{\prime \prime}, 748, \\
& \delta \Phi_{0}=+00^{\prime} 34^{\prime \prime}, 092 \div 01^{\prime} 48^{\prime \prime}, 709, \\
& \delta h=+0,9592621 R 10^{-2} \pm 0,1091774 R \quad 10 \div . \\
& \delta t_{0}=+2^{\prime}, 077 \pm 0^{\prime}, 4 .
\end{aligned}
$$

I valori più probabili delle coordinate spazio-temporali, o meglio, i 
Tabella II

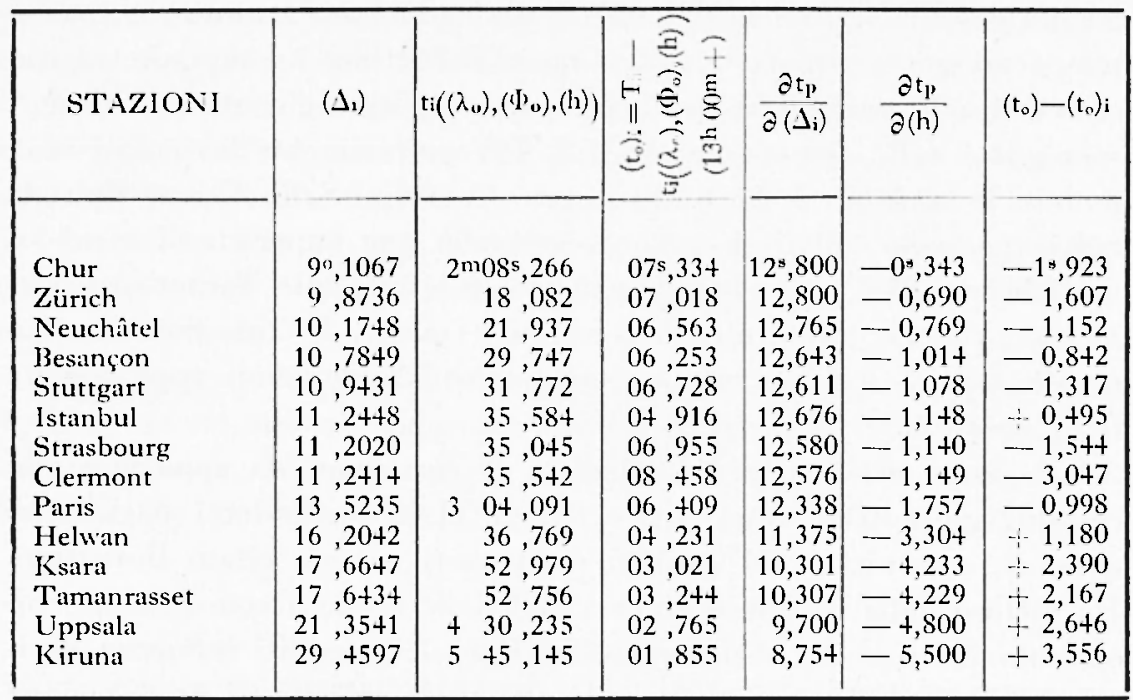

valori più adattalili, conformemente al metodo dei minimi quadrati, a: dati sperimentali utilizzati, sono pertanto:

$$
\left\{\begin{aligned}
\lambda_{\mathrm{o}} & =+14^{\prime} 53^{\prime} 41^{\prime \prime}, 916 \pm 02^{\prime} 04^{\prime \prime}, 748, \\
\Phi_{\mathrm{o}} & =+38^{\prime \prime} 25^{\prime} 16^{\prime \prime}, 193 \pm 01^{\prime} 48^{\prime \prime}, 709, \\
h & =(3,9592621 \pm 0,1091774) R \quad 10^{-2}+33 \mathrm{~km}=[1] \\
& =283,938 \mathrm{~km}=6,920 \mathrm{~km}, \\
t_{\mathrm{o}} & =13^{\mathrm{h}} 00^{\mathrm{m}} 07^{\circ}, 488+0^{\circ}, 4 .
\end{aligned}\right.
$$

I valori ottenuti si adattano in modo più che soddisfacente ai tempi di registrazione delle $P_{n}$ in quasi tutte le stazioni la cui distanza epicentrale è compresa tra 700 e $3000 \mathrm{~km} \mathrm{ca.} \mathrm{Si} \mathrm{avrebbe} \mathrm{invece} \mathrm{uno}$ scarto eccezionale per le stazioni più vicine all'epicentro, nel senso che tali stazioni avrebbero registrato con notevole anticipo l'onda $P_{n}$. Tale anticipo risulterebbe di 9",4 a Messina, di 8,7 a Regrio Calabria, di $12^{\circ}$ a Taranto (tabella III).

La notevole concordanza con i dati di stazione di alta fiducia come Chur, Zürich, Neuchâtel, Stuttgart, Strasbourę, Parigi, Uppsala, Kiruna, indurrebbe a considerare i risultati ottenuti come sufficientemente sicuri. Gli anticipi osservati nelle stazioni di Messina, Reggio Calabria 
e Taranto, pur non costituendo un fatto nuovo, in quanto anticipi dello stesso ordine sarebhero stati osservati, in analoghe circostanze, da Demetreseu, condurrebbero a conseguenze eccezionali. Ad esempio, l'onda $P_{11}$ avrebbe percorso il tratto ipocentro-stazione di Messina con una velociti media non inferiore a $10 \mathrm{~km} / \mathrm{sec}$, cioè, con una velocità media dello stesso ordine della velocità vera che, secondo Jeffreys $\left({ }^{4}\right)$, compete all'onda $P$ alla profondità di $600 \mathrm{~km}$.

In seguito a tali risultati, ritenendo indispensabile un controllo più rigroroso del materiale sperimentale fin qui utilizzato, ho inoltrato la richiesta dei sismogrammi presso vari Osservatori. La richiesta è stata gentilmente accolta ed in massima parte soddisfatta col sollecito invio di registrazioni originali o copie fotografiche.

Per quanto riguarda le stazioni, i cui dati sono stati utilizzati nel

TABella III

\begin{tabular}{|c|c|c|c|c|}
\hline STAZIONI & $\Delta$ & $\begin{array}{c}t_{p} \\
\text { (C) }\end{array}$ & $\begin{array}{l}t_{p} \\
(O)\end{array}$ & $\mathrm{O}-\mathrm{C}$ \\
\hline Reggio Calabria & $0^{\prime \prime}, 77+2$ & $00^{\mathrm{m}} 38^{\mathrm{s}}, 0$ & $00^{\mathrm{m}} 28^{\mathrm{s}}, 5$ & $-9^{\mathrm{s}, 5}$ \\
\hline Messina & 0,6622 & 37,5 & 28,8 & $-8,7$ \\
\hline Taranto & 2,6078 & 51,2 & 38,5 & $-12,7$ \\
\hline Rocca di Papa & 3,5602 & $01 \quad 00,9$ & 59,5 & $-1,4$ \\
\hline Roma & 3,7609 & 03,2 & $01 \quad 01,5$ & $-1,7$ \\
\hline Firenze Xim. & 5,8465 & 27,6 & 25,5 & $-2,1$ \\
\hline Prato & 5,9943 & $29,+$ & 28,5 & $-0,9$ \\
\hline Athene & 6,9670 & $+1,4$ & $3+, 9$ & $-6,5$ \\
\hline Trieste & 7,0800 & $+2,8$ & $+2,5$ & $-0,3$ \\
\hline Salò & 7,7100 & 50,6 & $+7,5$ & $-3,1$ \\
\hline Chur & 9,1279 & $02 \quad 08,2$ & $02 \quad 08,1$ & $-0,1$ \\
\hline Wien & 9,6936 & 15,2 & 15,5 & $\begin{array}{r}+3 \\
+\quad 1,3\end{array}$ \\
\hline Zürich & 9,8973 & 17,8 & 17,6 & $-0,2$ \\
\hline Neuchâtel & 10,2063 & 21,6 & 21,0 & $-0,6$ \\
\hline Besançon & 10,8187 & 29,2 & 28,5 & $-0,7$ \\
\hline Stuttgart & 10,9598 & 30,9 & 31,0 & $+0,1$ \\
\hline Istanbul & 11,1709 & 33,6 & 33,0 & $-0,6$ \\
\hline Strasbourg & $11,22+7$ & $3+, 2$ & 34,5 & $+0,3$ \\
\hline Clermont & 11,2892 & 35,0 & 36,5 & $\div \quad 1,5$ \\
\hline Praha & $11,+635$ & 37,2 & 40,5 & $\begin{array}{r}+3,3 \\
\end{array}$ \\
\hline Paris & 13,5609 & $\begin{array}{ll}03 & 02,9\end{array}$ & $03 \quad 03,0$ & $-\quad 0,1$ \\
\hline Lecle & 14,2859 & 11,6 & 11,5 & $-0,1$ \\
\hline Helwan & $16,1+27$ & 32,9 & 33,5 & $-0,6$ \\
\hline Ksara & 17,5928 & 48,2 & 48,5 & $+\quad 0,3$ \\
\hline Tamanrasset & 17,6883 & $+9,2$ & $+8,5$ & $-0,7$ \\
\hline Rathfarnham & 20,6838 & $04 \quad 19,1$ & $0+\quad 24,5$ & $-5,4$ \\
\hline Lippsala & 21,3397 & 25,5 & 25,5 & 0,0 \\
\hline Kiruna & $29,4+47$ & $05 \quad 39,7$ & $05 \quad 39,5$ & $-0,2$ \\
\hline
\end{tabular}


calcolo precedente (escludendo le stazioni Ksara ed Istanluul per mancato invio delle registrazioni), ho potuto notare:

1) gli inizi direttamente osservati sui sismogrammi coincidono in genere con gli inizi riportati nel Bollettino del B.C.I.S.; solo in alcuni casi si ha un divario di qualche decimo di secondo, dovuto certamente ad un criterio di arrotondamento adottato dall'osservatore;

2) sulla componente verticale a breve periodo di Clermont Ferrand, nonostante la lieve agitazione microsismica, si può individuare chiaramente l'inizio delle $P_{11}$ in una emersio alle $13^{11} 02^{\mathrm{m}} 42^{*}, 5$ che precede l'impetus rilevato dall'osservatore di $1^{\text {s }}, 5$.

Per quanto riguarda le altre stazioni ho notato:

1) l'inizio delle $P_{\mathrm{n}}$ è alle $13^{\mathrm{h}} 00^{\mathrm{m}} 37^{\mathrm{s}}$ a Reggio Calabria, alle $13^{\mathrm{h}} 00^{\mathrm{m}} 52^{\mathrm{s}}$ a Taranto, alle $13^{\mathrm{h}} \cdot 01^{\mathrm{m}} 45^{\mathrm{s}}$ ad Atene, per cui gli anticipi osservati in tali stazioni si ridurreblsero a 8:5 per Reggio Calabria, a 6*7 per Taranto, a 3 ,9 per Atene;

2) sul sismogramma inviato dalla stazione di Rathfarnham, nella forte agitazione microsismica, non è rilevabile alcun inizio senza ambiguitì.

\section{Tabella IV}

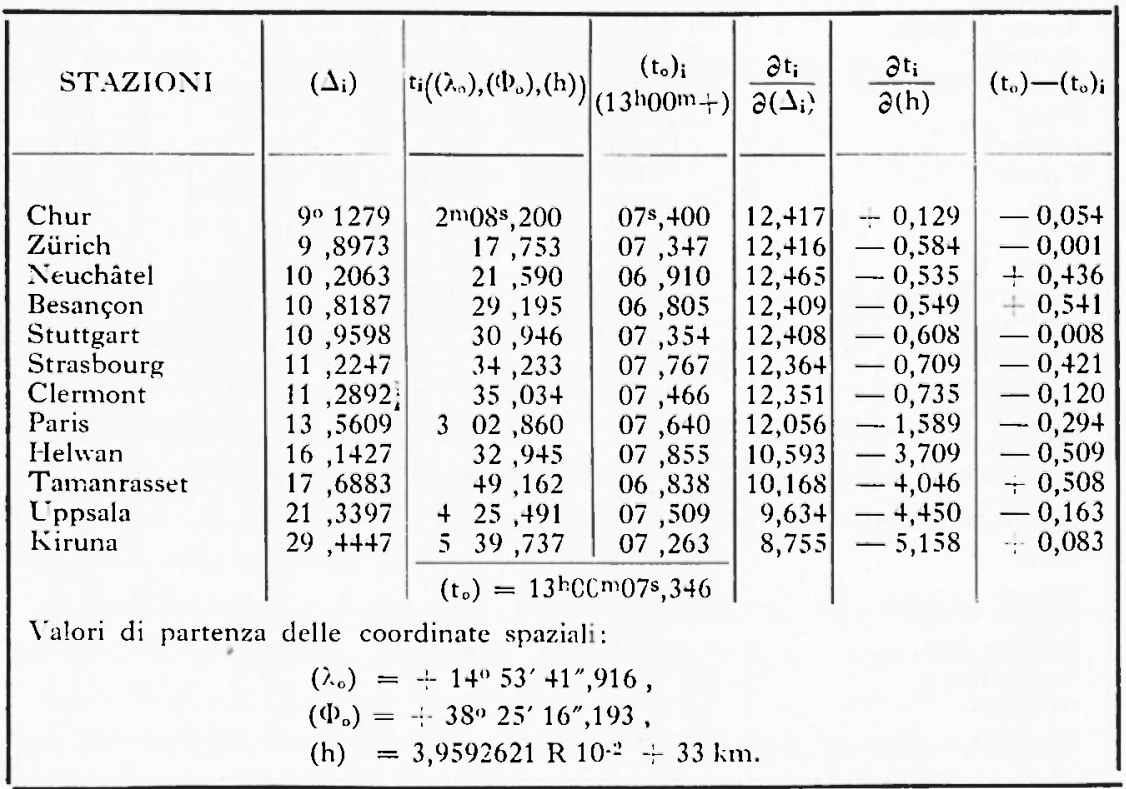


Il controllo effettuato confermerebbe sempre più $i$ risultati precedenti. Comunque, data la notevole correzione di profondità in precedenza ottenuta, ho ritenuto opportuno procedere ad una seconda approssimazione, servendomi dei dati direttamente osservati ed escludendo le stazioni di Istanbul e Ksara (tabella IV).

I risultati ottenuti sono i seguenti:

$$
\left\{\begin{array}{l}
\lambda_{\mathrm{o}}=+14^{\prime \prime} 49^{\prime} 46^{\prime \prime}, 391 \pm 01^{\prime} 45^{\prime \prime}, 788, \\
\Phi_{\mathrm{w}}=+38^{\prime \prime} 24^{\prime} 28^{\prime \prime}, 302 \pm 00^{\prime} 55^{\prime \prime}, 687 . \\
h=4,0113751 R \quad R \quad 10^{\circ}+33 \mathrm{~km}=0,0660169 R \quad 10^{\circ} . \\
t_{\mathrm{o}}=13^{\prime \prime} 00^{\mathrm{m}} 07^{\circ}, 535+0,249 .
\end{array}\right.
$$

Come somma dei quadrati dei residui bo ottenuto il valore:

$$
[v v]=[l .4]=0,886+822 .
$$

Esaminando la tabella (V) si può constatare che, riferendosi ai valori [2], resta più che confermato sia l'accordo con i dati delle stazioni con distanze epicentrali comprese tra 700 e $3000 \mathrm{~km}$ ca, sia il forte anticipo nelle stazioni di Messina, Reggio Calabria e Taranto. Pur volendo prescindere da quest'ultima circostanza, i valori [2] non possono essere accettati senza riserve.

Innanzi tutto l'entità degcli errori medi ottenuti indurrebbe ad attribuire ai valori calcolati un grado di precisione indubbiamente lontano dalle possililitì sperimentali.

Inoltre, tracciando le curve che rappresentano i tempi di tragitto delle $P$ in funzione della variabile $h$, in corrispondenza a diversi valori del parametro $\perp$ iquali risultano dalle dromocrone di J-B, che qui sono state sistematicamente assunte come dromocrone di riferimentol, si nota come tali curve abbiano, nell'intorno del punto $h=0,04 R+33 \mathrm{~km}, \quad$ un andamento rapidamente crescente con $h$ per piccoli valori di $\lrcorner$, praticamente stazionario per valori di $J$ compresi nell intervallo $7^{\prime \prime}-13^{\prime \prime}$, decisamente decrescente per valori di $\perp$ più elevati. Più precisamente, considerando l'andamento medio nell'intervallo $7^{\circ}-13^{\circ}$ della funzione $\frac{\hat{\partial} t_{\mathrm{P}}}{\partial h_{\mathrm{h}}}$, che, per $h=0,04 R+33 \mathrm{~km}$ (valore assai vicino al valore di partenza assunto nell'ultima determi- 


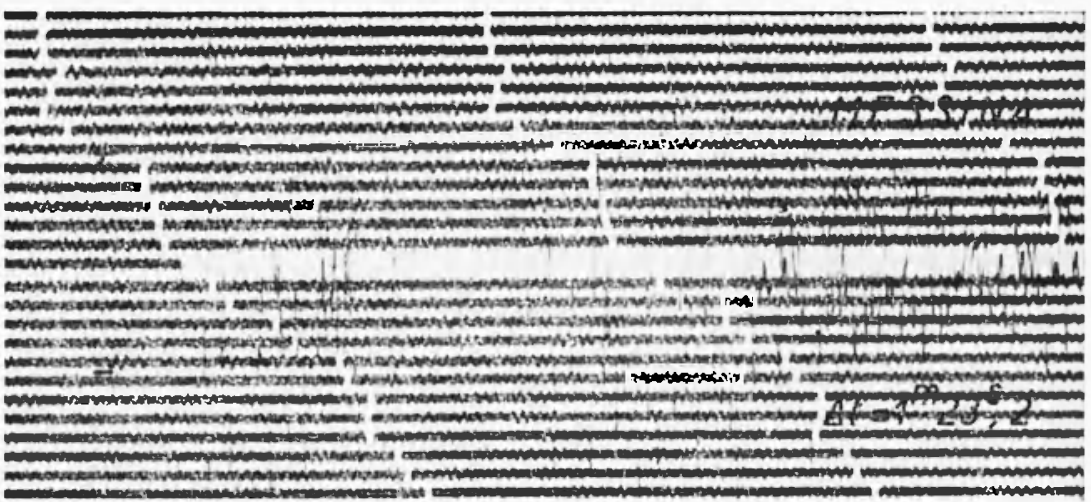

Fi匹. 1 Messina

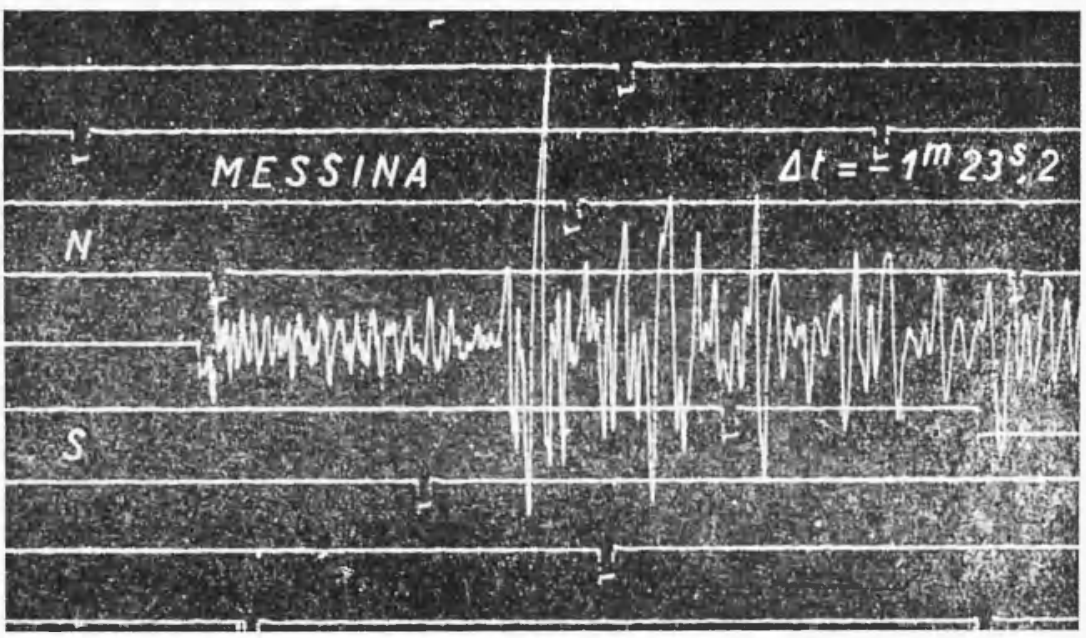

Fig. 2

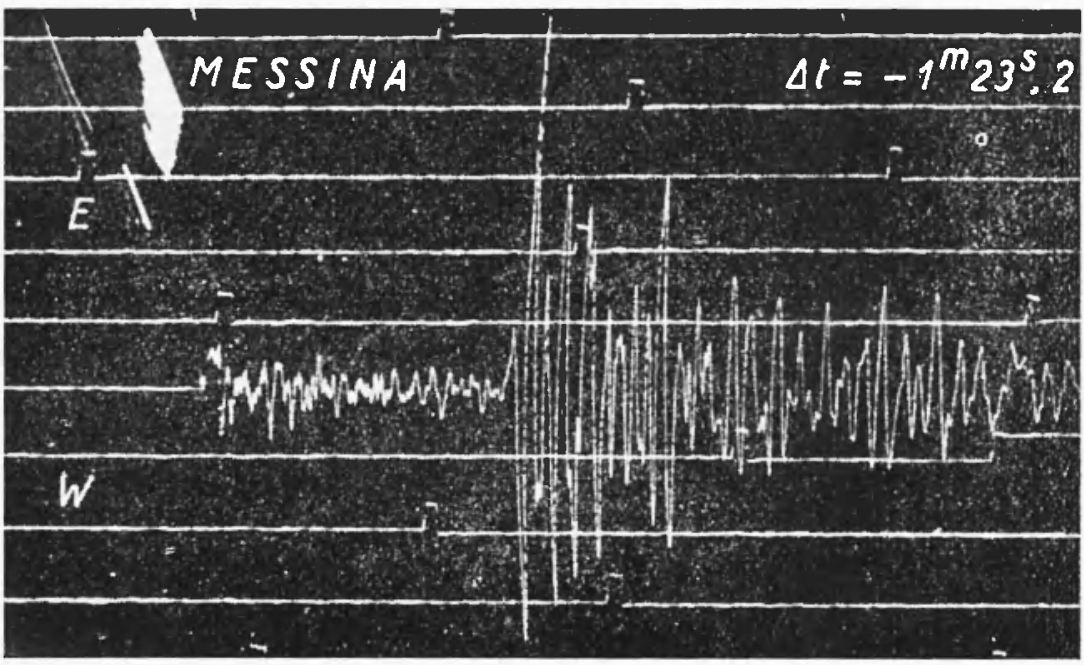

Fig. 3 
Tabella V

\begin{tabular}{|c|c|c|c|c|}
\hline STAZIONI & $\Delta$ & $\begin{array}{l}{ }^{t_{p}} \\
\text { (C) }\end{array}$ & $\begin{array}{l}t_{p} \\
(O)\end{array}$ & $\mathrm{O}-\mathrm{C}$ \\
\hline Messina & $0 \bullet, 6956$ & $00 n 38^{s}, 0$ & $00 \mathrm{~m} 28 s, 8$ & $-9 \mathrm{~s} 2$ \\
\hline Reggio Calabria & 0,8060 & $38,+$ & 29,5 & $-8,9$ \\
\hline 'Tariunto & 2,6529 & 51,9 & $4+, 5$ & $-7,+$ \\
\hline Rocca di Papa & $3,5+89$ & 0101,0 & 59,5 & $-1,5$ \\
\hline Roma & $3,7+86$ & 03,2 & $01 \quad 01,3$ & $-1,9$ \\
\hline Firenze Xim. * & 5,8356 & 27,6 & 25,5 & $-2,1$ \\
\hline Prato * & 5,9829 & 29,3 & 28,5 & -0.8 \\
\hline Athene & 7,0189 & $+2,1$ & 37,5 & $-4,6$ \\
\hline 'Trieste & 7,0876 & $+2,9$ & $+2,5$ & $-0,4$ \\
\hline Salò & 7,7019 & 50,5 & 47,5 & $-3,0$ \\
\hline Chur & 9,1195 & $02 \quad 08,1$ & $02 \quad 08,1$ & 0,0 \\
\hline Wien * & 9,7122 & $15,+$ & 15,5 & $-0,1$ \\
\hline Zürich & 9,8870 & 17,6 & 17,6 & 0,0 \\
\hline Neuchâtel & 10,1902 & $21,+$ & 21,0 & $-0,+$ \\
\hline Besançon & 10,8010 & 28,9 & 28,5 & $\div 0,+$ \\
\hline Stuttgart & $10,95+6$ & 30,8 & 30,8 & 0,0 \\
\hline Istanbul * & 11,2238 & $3+, 2$ & 33,0 & $\div 0,2$ \\
\hline Strasbourg & 11,2151 & $3+, 1$ & $3+, 5$ & $-0,+$ \\
\hline Clermont & 11,2609 & $3+, 6$ & 35,0 & $-0,+$ \\
\hline Praha & $11,+755$ & 37,3 & 39,6 & $+2,3$ \\
\hline Paris & $13,5+05$ & $03 \quad 02,5$ & $03 \quad 03,0$ & $\div 0,5$ \\
\hline Uccle & $1+, 2736$ & 11,4 & 11,5 & $+0,1$ \\
\hline Helwan & 16,1819 & 33,2 & 33,5 & $+0,3$ \\
\hline Ksara * & $17,6+13$ & 48,5 & 48,5 & 0,0 \\
\hline Tamanrasset & 17,6514 & 48,6 & 48,5 & -0.1 \\
\hline Lppsala & 21,3564 & $0+25,+$ & 25,5 & $-0,1$ \\
\hline Kiruna & $29,+617$ & $05 \quad 39,6$ & 39,5 & $-0,1$ \\
\hline
\end{tabular}

nazione), puo essere espressa con huona approssimazione dalla relazione:

$$
\left.\frac{\partial t_{\mathrm{P}}}{\partial h}=6.337-0,8601515 د+0.0204545\right\lrcorner^{2}
$$

si nota come, nell'intorno del valore $\lrcorner=10^{\circ}$, tale funzione assume valori che si scostano assai poco dal valore zero. Se si tiene presente che il metodo più adoperato è basato sulla soluzione del sistema di equazioni di condizione

$$
\begin{aligned}
& \delta t_{0}-\frac{\partial t_{p}}{\partial\left(\Delta_{i}\right)} \cos \left(\Phi_{0}\right) \operatorname{sen}\left(\alpha_{i}\right) \delta \lambda_{0}-\frac{\partial t_{p}}{\partial\left(\Delta_{i}\right)} \cos \left(\alpha_{i}\right) \tilde{\delta} \Phi_{0}+\frac{\partial t_{p}}{\partial(h)} \delta h+ \\
& -\left\{T_{i}-\left[\left(\iota_{0}\right)+\iota_{\mathrm{p}}\left(\left(\lambda_{\mathrm{o}}\right),\left(\Phi_{\mathrm{o}}\right),(h)\right)\right]=v_{\mathrm{i}} \quad(i=1,2, \ldots, n),\right.
\end{aligned}
$$


dedotte in corrispondenza a ciascuna delle $n$ stazioni di osservazione, si deduce che il gruppo di equazioni associate a quelle stazioni la cui distanza cade nell'intorno considerato non dà un contributo apprezzabile nella determinazione $\mathrm{di} \delta h$, in quanto le equazioni di tale gruppo, anche per valori sensibilmente diversi di $\delta h$, possono dar luo. go a residui che praticamente possono essere mascherati dai residui dovuti agli errori di osservazione.

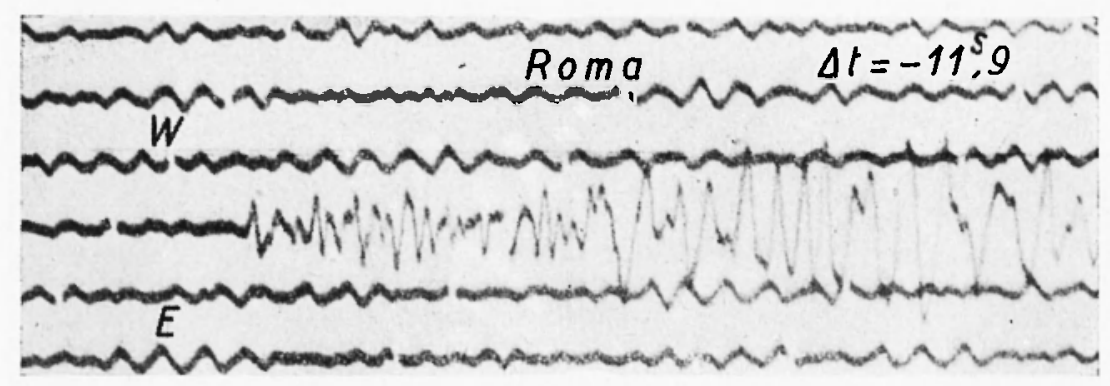

Fig. 4

Nell'ultima approssimazione eseguita sono stati utilizzati dati di stazioni le cui distanze sono in prevalenza comprese nello intervallo critico considerato. I soli dati di Helwan, Tamanrasset, Uppsala e Kiruna, che, ai fini della valutazione di $\delta / h$ possono essere considerati sensibilmente determinativi, non sono statisticamente sufficienti.

Successivamente ho avuto la possibilità di esaminare i sismogrammi di College, Hungry Horse, Tucson ed Isabella, inviatimi dall'U.S.C.G.S. e dal Prof. Gutenberg. Gli inizi, rilevabili con sufficiente precisione, darebluero luogo, in base alla precedente determinazione, alle seguenti differenze $\mathrm{O}-\mathrm{C}$ :

$\begin{array}{ll}\text { College } & +3^{\mathrm{s}, 4} \\ \text { Hungry Horse } & +4,0 \\ \text { Tucson } & +5,2 \\ \text { Isabella } & +4,9,\end{array}$

per cui tali stazioni avrebbero registrato l'onda $P$ con un sensibile ritardo. Ho ravvisato pertanto l'opportunità di eseguire una ulteriore 


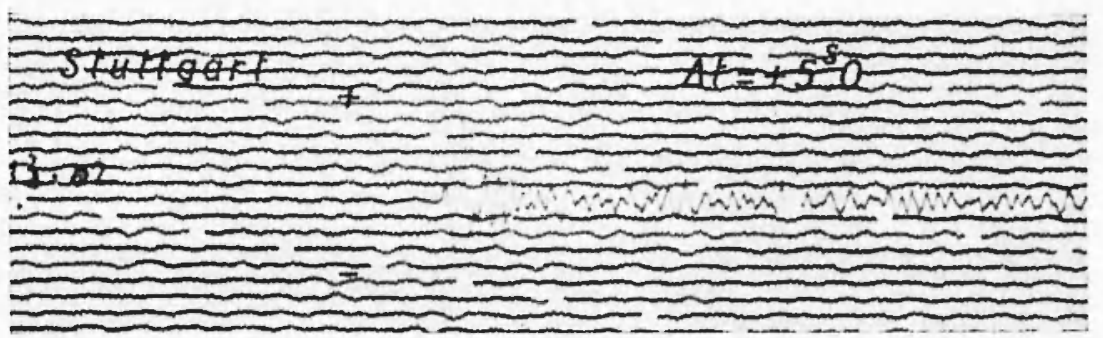

Fig. 5

19 s.

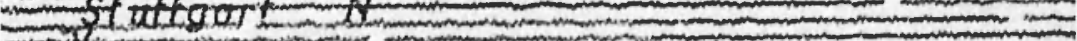
Crin

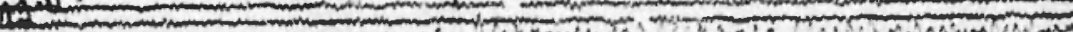
o.

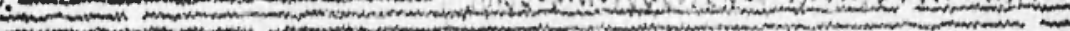

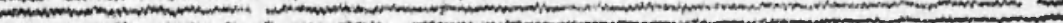
M 10

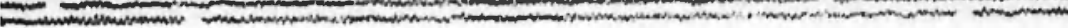

Fig. 6

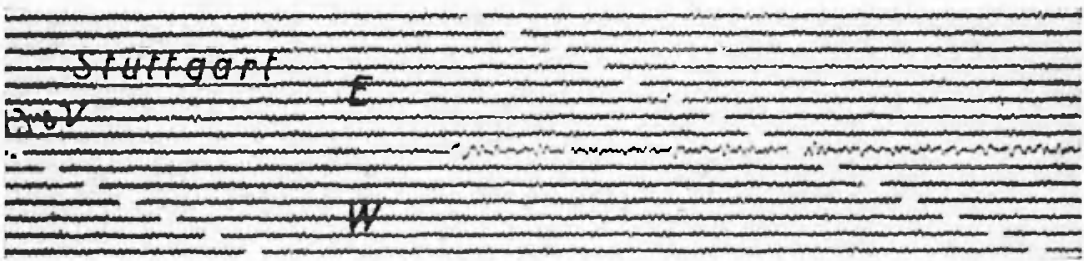

Fig. ?

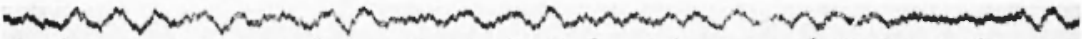
N

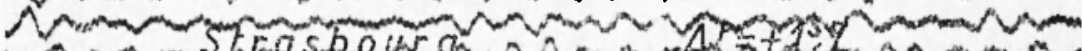

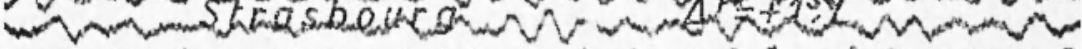

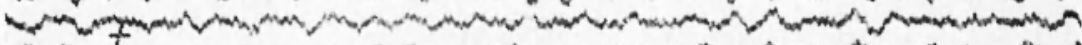

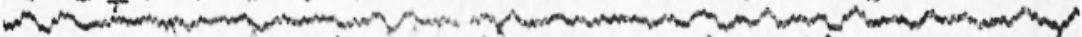

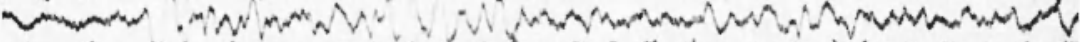

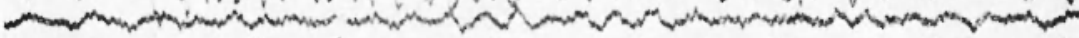

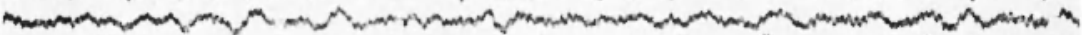

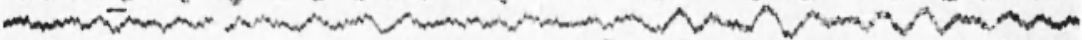

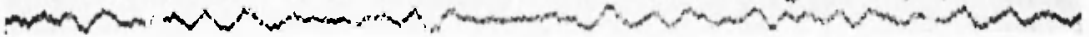

Fig. 8 
determinazione utilizzando anche gli inizi osservati nelle stazioni lontane ed insistendo nel proposito di escludere i dati delle stazioni vicine.

I dati sperimentali utilizzati, unitamente agli elementi essenziali per il calcolo, sono contenuti nella tabella VI.

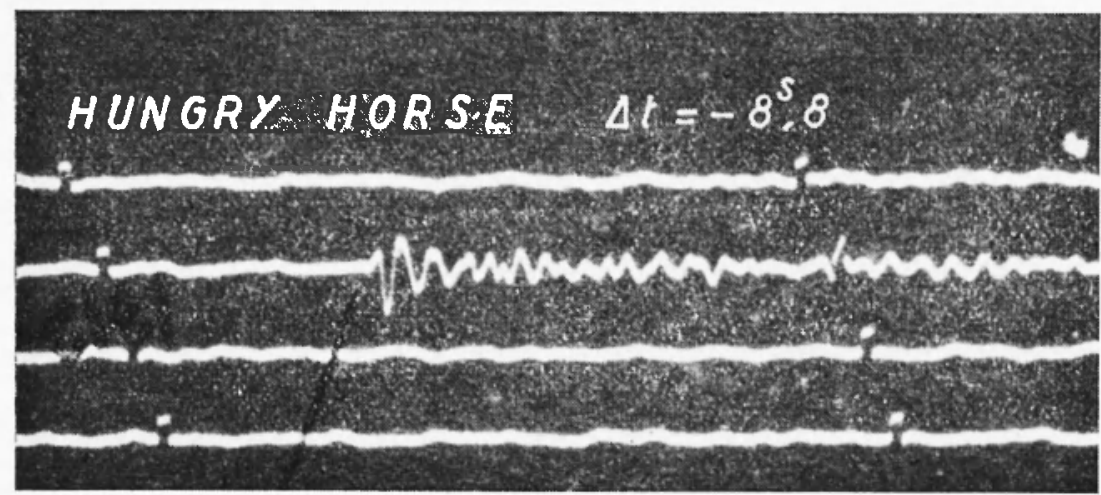

Fig. 9

Le correzioni ottenute sono le seguenti:

$$
\begin{aligned}
& \delta \lambda_{\mathrm{o}}=+r^{\prime}+\mathrm{l}^{\prime \prime} .660-5^{\prime}+\mathrm{l}^{\prime \prime} .223 \text {. } \\
& \delta \Phi_{\mathrm{o}}=-3^{\prime} 25^{\prime \prime}, 81 \tau \div 3^{\prime} 17^{\prime \prime}, 331 \text {, } \\
& \delta h=-0.7051056 R \quad 10^{-2}=0,1249318 R \quad 10^{\circ 2} \text {. } \\
& \delta t_{\mathrm{o}}=-1 \mathrm{1}, 986=0 \times, 726 \text {, }
\end{aligned}
$$

$\operatorname{con}$

$$
[v v]=[l l, 4]=16,3253473
$$

per cui i valori delle coordinate spazio-temporali risultano:

$$
\left\{\begin{aligned}
\lambda_{\mathrm{o}} & =+15^{\prime} 01^{\prime} 23^{\prime \prime}, 576=5^{\prime} 41^{\prime \prime}, 223 \\
\Phi_{\mathrm{o}} & =+38^{\prime \prime} 21^{\prime} 50^{\prime \prime}, 376-3^{\prime} 17^{\prime \prime}, 331, \\
\varphi_{\mathrm{o}} & =+38^{\prime \prime} 33^{\prime} 09^{\prime \prime}, 059=3^{\prime} 17^{\prime \prime}, 331 \text { (lat. geografica } . \\
h & =3,2541566 R 10^{\circ}+33 \mathrm{~km}=0,1249318 R \quad 10^{\circ 2}= \\
& =239,248 \mathrm{~km}=7,918 \mathrm{~km} \\
t_{\mathrm{u}} & =13^{\mathrm{h}} 00^{\mathrm{m}} 05^{\mathrm{s}}, 5=0^{\prime}, 7 .
\end{aligned}\right.
$$


Tabella VI

\begin{tabular}{|c|c|c|c|c|c|c|c|}
\hline STAZIONI & $\left(\Delta_{\mathrm{i}}\right)$ & $\begin{array}{c}\mathrm{T}_{\mathrm{i}} \\
\left(13^{\mathrm{h}} \div\right)\end{array}$ & $t_{i}\left(\left(\lambda_{u}\right),\left(W_{0}\right),(h)\right)$ & $\begin{array}{c}\left(t_{c}\right)_{i} \\
(13 h 00 m+r)\end{array}$ & $\frac{\partial t_{p}}{\partial\left(\Delta_{i}\right)}$ & $\frac{\partial t_{p}}{\partial(h)}$ & $\left(t_{0}\right)-\left(t_{11}\right)_{i}$ \\
\hline Chur & 90,1279 & $02^{m} 15^{s}, 6$ & $02^{\mathrm{m} 08^{\mathrm{s}}, 200}$ & $07 s, 400$ & $12,+17$ & 0,129 & $+0,088$ \\
\hline Zürich & 9,8973 & 25,1 & 17,753 & $07,3+7$ & $12,+16$ & $-0,584$ & $+0,1+1$ \\
\hline Neuchatel & 10,2063 & 28,5 & 21,590 & 06,910 & $12,+65$ & $-0,535$ & $+0,578$ \\
\hline Besançon & 10,8187 & 36,0 & 29,195 & 06,805 & 12,409 & $-0,549$ & $+0,683$ \\
\hline Stuttgart & 10,9598 & 38,3 & $30,9+6$ & 07,354 & $12,+08$ & $-0,608$ & $+0,134$ \\
\hline Strasbourg & 11,2247 & $+2,0$ & $3+, 233$ & 07,767 & $12,36+$ & $-0,709$ & $-0,279$ \\
\hline Clermont & 11,2892 & 42,5 & 35,034 & $07,+66$ & 12,351 & $-0,735$ & $\dashv-0,022$ \\
\hline Paris & 13,5609 & $03 \quad 10,5$ & $03 \quad 02,860$ & 07,640 & 12,056 & $-1,589$ & $-0,252$ \\
\hline Helwan & $16,1+27$ & 40,8 & $32,9+5$ & 07,855 & 10,593 & $-3,709$ & $-0,367$ \\
\hline Tamanrasset & 17,6883 & 56,0 & 49,162 & 06,838 & 10,168 & $-4,046$ & $+0,650$ \\
\hline Uppsala & 21,3397 & $0+33,0$ & $0+25,491$ & 07,509 & 9,634 & $-4,405$ & $-0,021$ \\
\hline Kiruna & $29,4+47$ & $05+7,0$ & $05 \quad 39,737$ & 07,263 & 8,755 & $-5,158$ & $+0,225$ \\
\hline College & 75,9802 & 1128,1 & $11 \quad 17,+66$ & 10,634 & 5,502 & $-6,512$ & $-3,1+6$ \\
\hline Hungry I Iorse & $82,26+4$ & 1202,0 & 50,931 & 11,069 & 5,076 & $-6,639$ & $-3,581$ \\
\hline Tucson & $93,308+$ & 56,0 & $12+3,773$ & 12,227 & 4,531 & $-6,808$ & $-4,339$ \\
\hline \multirow[t]{2}{*}{ Isabella } & $94,+569$ & 1301,0 & 48,987 & 12,013 & 4,554 & $-6,808$ & $-4,525$ \\
\hline & \multicolumn{3}{|c|}{$\begin{aligned}\left(\lambda_{0}\right) & =+14^{\circ} 53^{\prime}+1^{\prime \prime}, 916 \\
\left(\Phi_{0}\right) & =+38^{\circ} 25^{\prime} 16^{\prime \prime}, 193\end{aligned}$} & & \multicolumn{2}{|c|}{$+33 \mathrm{~km}$} & \\
\hline
\end{tabular}


Nella tabella VII sono riportati: le distanze epicentrali, i corrispondenti tempi di tragitto delle $P$ dedotti dalle tabelle di J-B $(C)$, i tempi di tragitto osservati $(O)$ e le differenze $O-C$. quali risultano dall'ultima determinazione effettuata. Si può notare come l'intervento

\section{TABELla VII}

\begin{tabular}{|c|c|c|c|c|c|}
\hline STAZIOXI & 1 & $\mathrm{~T}_{\mathrm{p}}$ & $\begin{array}{c}t_{p} \\
\text { (C) }\end{array}$ & $\begin{array}{c}t_{p} \\
(O)\end{array}$ & $\mathrm{O}-\mathrm{C}$ \\
\hline Messina & $0^{\circ}, 5483$ & $13 \mathrm{~h} 00 \mathrm{~m} 36^{\mathrm{s}}, 3$ & $00 \mathrm{~m} 32 \mathrm{~s}, 0$ & $00^{\mathrm{m}} 30^{\mathrm{s}, 8}$ & $-1 \mathrm{~s}, 2$ \\
\hline Reggio Calabria & 0,6606 & 37,0 & 32,5 & 31,5 & $-1,0$ \\
\hline Taranto & 2,5821 & 52 & $+7,8$ & 46,5 & $-1,3$ \\
\hline Rocca di Papa & 3,6576 & 0107 & 59,9 & 0101,5 & $+1,7$ \\
\hline Roma & 3,8592 & 08,8 & $01 \quad 02,2$ & 03,3 & $+1,1$ \\
\hline Firenze Xim. * & $5,9+34$ & 33 & 27,9 & 27,5 & $-0,4$ \\
\hline Prato & 6,0915 & 36 & 29,8 & 30,5 & 0,7 \\
\hline Athene & 6,8644 & 45 & 39,6 & 39,5 & $-0,1$ \\
\hline Trieste & 7,1488 & 50 & $+3,2$ & 44,5 & $+1,3$ \\
\hline Salò & 7,8028 & 55 & 51,8 & $+9,5$ & $-2,3$ \\
\hline Chur & 9,2210 & 0215,6 & $02 \quad 09,6$ & 0210,1 & $+0,5$ \\
\hline Wien * & 9,7408 & 23 & 16,2 & 17,5 & $+1,3$ \\
\hline Zürich & 9,9926 & 25,1 & 19,4 & 19,6 & $+0,2$ \\
\hline Neuchâtel & 10,3084 & 28,5 & 23,4 & 23,0 & $-0,4$ \\
\hline Besançon & 10,9226 & 36 & 31,2 & 30,5 & $-0,7$ \\
\hline Stuttgart & 11,0484 & 38,3 & 32,8 & 32,8 & 0,0 \\
\hline Istanbul * & 11,0919 & 40,5 & 33,4 & 35,0 & $-1,6$ \\
\hline Strasbourg & 11,3192 & $+2,0$ & 36,2 & 36,5 & $-0,3$ \\
\hline Clermont & 11,4017 & 42,5 & 37,3 & 37,0 & $-0,3$ \\
\hline Praha & 11,5236 & 47,1 & 38,8 & 41,6 & $+2,8$ \\
\hline Paris & 13,6673 & 0310,5 & 0305,4 & $03 \quad 05,0$ & $-0,4$ \\
\hline Uccle & $1+, 3837$ & 19,0 & $1+, 2$ & 13,5 & $-0,7$ \\
\hline Helwan & 16,0271 & 40,8 & 33,9 & 35,3 & $+1,4$ \\
\hline Ksara * & 17,4844 & 56 & 50,0 & 50,5 & $-0,5$ \\
\hline Tamanrasset & 17,6888 & 56 & 52,2 & 50,5 & $-1,7$ \\
\hline Uppsala & 21,3902 & $04 \quad 33$ & $0+29,4$ & $0+\quad 27,5$ & $-1,9$ \\
\hline Kiruna & $29,49+3$ & $05+7$ & $05+4,1$ & $05+1,5$ & $-2,6$ \\
\hline College & 76,0500 & 1128,1 & 1122,6 & 1122,6 & 0,0 \\
\hline Hungry I Iorse & 82,3657 & 1202 & 1156,3 & 56,5 & $+0,2$ \\
\hline Tucson & $93,+191$ & 56 & $12+9,2$ & 1250,5 & $-1,3$ \\
\hline Isabella & 94,5626 & 1301 & 54,4 & 55,5 & $-1,1$ \\
\hline
\end{tabular}

dei dati relativi alle quattro stazioni lontane di College, Hungry Horse, Tucson ed Isabella dà luogo ad una valutazione delle coordinate spaziotemporali che fa rientrare gli anticipi delle stazioni più vicine nei limiti degli scarti attribuibili, oltre che agli errori di osservazione, alle varie cause d'indeterminazione che in genere influenzano l'approssimazione con cui si può determinare la posizione di un ipocentro. Inoltre, non solo resta conservato, come del resto era prevedibile per 
le considerazioni già fatte, il buon accordo con i dati del gruppo di stazioni centro-europee, ma si ottiene una sufficiente concordanza con i tempi osservati nelle stazioni più lontane.

Messina - Osservatorio Geofisico dell Istituto Nazionale di Geofisica Settembre 1955.

\section{RIASSUNTO}

E noto che non is facile stabilire con precisione la profondita di un ipocentro. anche quando si ha la possibilità di utilizare chiari inizi di onde caratteristiche dei terremoti profondi come le $\mathrm{pP}, \mathrm{sP}, \mathrm{sS}$, ecc. Luso di laboriosi metodi statistici può, in condizioni favorevoli, condurre a risultati con una aprossimazione dellordine di $10 \mathrm{~km}$. Loperazione i resa ancora più ardua nel caso in cui lintensità del terremoto i talmente debole da non dar luogo, come nel caso in rame, alla registrazione di onde $\mathrm{PP}, \mathrm{sP}, \mathrm{s}, \ldots$ In queste condizioni sono utilizabili, se in numero sufficiente, gli inizi delle $\mathrm{P}$ nelle varie stazioni.

Lapplicazione di metodi statistici, fondati su un criterio di adattamento di tali inizi a dromocrone prestabilite, può dar luogo a risultati tanto più precisi quanto più attendibili sono le dromocrone di riferimento.

Le numerose applicazioni e gli ottimi risultati conseguiti dimostrano che il metodo di Caloi-Peronaci, associato all uso delle dromocrone di Jeffreys e Bullen, permette la contemporanea determinazione delle quattro coordinate spazio-temporali di un ipocentro profondo con notevole precisione. Partendo da uno stesso valore della profondità, con tale metodo sono stati qui effettuati due tentativi, il primo utilizando dati di stazioni le cui distanze epicentrali sono in prevalenza addensate nell intorno dei $10^{\prime \prime}$, il secondo facendo intervenire anche $i$ dati di quattro stazioni con distanza superiore a $75^{\circ}:$ i valori ottenuti per la profondità risultano notevolmente diversi. L'equazione fondamentale del metodo giustifica tale diversità e permette di escludere $i$ risultati ottenuti nel primo tentativo, in quanto conseguiti utilizzando prevalentemente dati relativi a distanze rispetto alle quali $i$ tempi di propagazione delle $\mathrm{P}$ sono poco sensibili alle variazioni di profondita. 
I valori ottenuti nellultima determinazione non mettono in evidenza anticipi apprezabili nei tempi di arrivo dellonda $\mathrm{P}_{\mathrm{n}}$ nelle stazioni più vicine allepicentro.

\section{$S U M M+A Y$}

It is well hnoun that it is not eassy to determine with exactness the depth of hypocentre, even when there is the possibility of utilizing the ovident beginnings of the waves which are characteristic for derp earthquates, litie the $\mathrm{PP}, \mathrm{SP}$, sS waves, etc. The use of laborious statistical methods can - in propitious conditions - give a result with an approximation of 10 hilometres. The operation is more difficult in case the intensity of the earthquaque is so weak that it does not cause - as in the case now discussed - the registration of $\mathrm{pP}, \mathrm{sP}, \mathrm{sS}$ waves. Under these circumstances, the beginnings of the $\mathrm{P}$ uaves registered in various observatories are utilizable, if in sufficient quantity.

The more veliable are the dromocrones reffered to - the move precise will be the results given by the application of statistical methods based on adaption principles of such beginnings on predetermined dromocrones.

The numerous applications and the excellent results obtained demonstrate that the metod Caloi-Peronaci, along with the use of Jeffreys and Bullen's dromocrones, allows - with remarkable exactness - the contemporary determination of four spacial-temporal coordinates of a deep hypocentre. Tuo attempts have been made with this method taking into consideration the same value of depth, the first one utilizing the data of those observatories, the epicentral distances of which are mostly amassed around the $10^{\circ}$, the second one with intervention of the data of four observatories at a distance greater than 75 : the values obtained regarding the depth differ considerably. The fondamental equation of the metod justifies such a diversity and allous the exclusion of the results obtained by the first attempt because these results uere obtained by utilizing in the main the data relating to distances in respert of which the propagation times of $\mathrm{P}$ waves are not sensitive enough to depth variations.

The values obtained in the last determination do not make evident considerable anticipations of time of arrival of the $\mathrm{P}_{11}$ uaves to the observatories which are neare to the epicentre. 


\section{BIBLIOGRAFIA}

(1) B. Gutenbers and C. F. Richter, Seismicity of the Earth and associated phenoment. Princeton University press, New Jersey (1949).

(2) D. Dr Filipe, Studio microsismico del terremolo del basso Tirreno del 16 marzo 1941. Boll. Soc. Sismol. It., vol. XXXIX, nn. 34. (1941).

(3) A. Girlanda, Studio di un movimento sismico del basso Tirreno, originante alla profonditì di $265 \mathrm{~km}$. Rend. Acc. Naz. dei Lincei, serie VIII, vol. XVIIl, 3 (1955).

(4) P. Caloi e F. Peroxici, Il terremolo del Turkestan del 2 novembre 1946. Annali di Geofisica, vol. I, n. 2 (19.48). 